\title{
On the geometry of the first and second Painlevé equations
}

\author{
Raouf Dridi \\ dridi.raouf@gmail.com \\ Doppler Institute for Mathematical Physics and Applied Mathematics, \\ Brehova 7, 11519 Prague 1, Czech Republic
}

March 3, 2022

\begin{abstract}
In this paper we explicitly compute the transformation that maps the generic second order differential equation $y^{\prime \prime}=f\left(x, y, y^{\prime}\right)$ to the Painlevé first equation $y^{\prime \prime}=6 y^{2}+x$ (resp. the Painlevé second equation $\left.y^{\prime \prime}=2 y^{3}+y x+\alpha\right)$. This change of coordinates, which is function of $f$ and its partial derivatives, does not exist for every $f$; it is necessary that the function $f$ satisfies certain conditions that define the equivalence class of the considered Painlevé equation. In this work we won't consider these conditions and the existence issue is solved on line as follows: If the input equation is known then it suffices to specialize the change of coordinates on this equation and test by simple substitution if the equivalence holds. The other innovation of this work lies in the exploitation of discrete symmetries for solving the equivalence problem.
\end{abstract}

\section{Introduction}

By fiber-preserving transformations we mean analytical transformations of the form

$$
\mathbb{C}^{2} \ni(x, y) \rightarrow(\bar{x}(x), \bar{y}(x, y)) \in \mathbb{C}^{2}
$$

with the condition $\bar{x}_{x} \bar{y}_{y} \neq 0$ expressing their local invertibility. These transformations form a Lie pseudogroup with

$$
\bar{x}_{y}=0, \bar{x}_{x} \bar{y}_{y} \neq 0
$$

as defining system.

As indicated in the abstract, our aim is to explicitly compute the transformation of this form that maps the second order equation

$$
\mathcal{E}_{f}: y^{\prime \prime}=f\left(x, y, y^{\prime}\right)
$$

where $y^{\prime}=\frac{d}{d x} y(x)$, to the first Painlevé equation (resp. to the second Painlevé equation). This change of coordinates, which is clearly function of $f$ and its partial derivatives, does not exist for every $f$; it is necessary that the function $f$ satisfies certain conditions that define the equivalence class of the considered Painlevé equation. Comparing to (KLS85) and (HD02), the existence issue is solved here on line as follows: If the input equation is known then it suffices to specialize the change of coordinates on this equation and test by simple substitution if the equivalence holds.

The calculations of transformation candidates are based on the following result (DP07). Given a Lie pseudo-group of transformations $\Phi$ and denote by $\mathcal{S}_{\mathcal{E}_{f}, \Phi}$ the symmetry pseudo-group of the equation $\mathcal{E}_{f}$ w.r.t to $\Phi$ i.e., $\mathcal{S}_{\mathcal{E}_{f}, \Phi}=\Phi \cap \operatorname{Diff}^{\text {loc }}\left(\mathcal{E}_{f}\right)$. In (DP07), we proved

(i) The number of constants appearing in the change of coordinates is exactly the dimension of $\mathcal{S}_{\mathcal{E}_{\bar{f}}, \Phi}$. This implies that when this dimension vanishes the change of coordinate can be obtained without integrating differential equations. Also, we have $\operatorname{dim}\left(\mathcal{S}_{\mathcal{E}_{f}, \Phi}\right)=\operatorname{dim}\left(\mathcal{S}_{\mathcal{E}_{\bar{f}}, \Phi}\right)$.

(ii) In the particular case when $\operatorname{dim}\left(\mathcal{S}_{\mathcal{E}_{\bar{f}}, \Phi}\right)=0$, the transformation $\varphi$ is algebraic in $f$ and its partial derivatives and it is obtained without solving differential equations. The degree of this transformation $\varphi$ is exactly equal to the finite value $\operatorname{card}\left(\mathcal{S}_{\mathcal{E}_{\bar{f}}, \Phi}\right)$. 
The last case is exactly what happens when $\mathcal{E}_{\bar{f}}$ is one of the Painlevé equations and $\Phi$ is the pseudogroup of fiber-preserving transformations or more generally point transformations. Indeed, the classical Lie analysis shows that the point symmetry pseudo-group of each one of Painlevé equations is zerodimensional. Moreover, according to the fact that the unique transformations that preserve the singularity structure are the homographic transformations, one can show by straightforward computations that the point symmetry pseudo-group of Painlevé one is

$$
\left\{\begin{array}{l}
\bar{x}=x \frac{\bar{y}^{2}}{y^{2}}, \\
\bar{y}^{5}=y^{5},
\end{array}\right.
$$

and

$$
\left\{\begin{array}{l}
\bar{x}=x \frac{\bar{y}^{2}}{y^{2}}, \\
\bar{y}^{3}=\frac{\bar{\alpha}}{\alpha} y^{3}, \\
\bar{\alpha}^{2}=\alpha^{2},
\end{array}\right.
$$

for Painlevé two when $\alpha \neq 0$ and

$$
\left\{\begin{array}{l}
\bar{x}=x \frac{\bar{y}^{2}}{y^{2}}, \\
\bar{y}^{6}=y^{6},
\end{array}\right.
$$

when $\alpha=0$.

Fiber-preserving transformations are suited when dealing with Painlevé equations. In particular, such transformations preserve the integrability in the sense of Poincaré (CM08). However, since Painlevé equations lie in the class of equations of the form

$$
y^{\prime \prime}=A(x, y)+B(x, y) y^{\prime}+C(x, y) y^{\prime 2}+D(x, y) y^{\prime 3}
$$

which is invariant under point transformation 1 , we consider in the last section of this paper the equivalence under these more general transformations.

\section{Building the invariants}

Le $\left(x, y, p=y^{\prime}\right)$ be a system of local coordinates of $\mathrm{J}^{1}=\mathrm{J}^{1}(\mathbb{C}, \mathbb{C})$, the space of first order jets of functions $\mathbb{C} \ni x \rightarrow y(x) \in \mathbb{C}(\underline{\text { Olv93 }})$. Two scalar second order ordinary equations

$$
\mathcal{E}_{f}: y^{\prime \prime}=f\left(x, y, y^{\prime}\right) \text { and } \mathcal{E}_{\bar{f}}: \bar{y}^{\prime \prime}=\bar{f}\left(\bar{x}, \bar{y}, \bar{y}^{\prime}\right)
$$

are said to be equivalent under a point transformation $\varphi$ if its first prolongation (to $\mathrm{J}^{1}$ ) maps the contact forms

$$
\left\{\begin{aligned}
\omega^{1} & =d y-p d x \\
\omega^{2} & =d p-f(x, y, p) d x
\end{aligned}\right.
$$

to the contact forms

$$
\left\{\begin{aligned}
\bar{\omega}^{1} & =d \bar{y}-\bar{p} \overline{d x} \\
\bar{\omega}^{2} & =d \bar{p}-\bar{f}(\bar{x}, \bar{y}, \bar{p}) d \bar{x}
\end{aligned}\right.
$$

up to an invertible $2 \times 2$-matrix of the form

$$
\left(\begin{array}{cc}
a_{1} & 0 \\
a_{2} & a_{3}
\end{array}\right)
$$

\footnotetext{
${ }^{1}$ Indeed, as remarked by E. Cartan (Car24), the above equation can always be regarded as the geodesics equation of a projective structure on a surface with local coordinates $x$ and $y$ and thus invariant under point transformations.
} 
The $a_{i}$ are functions from $J^{1}$ to $\mathbb{C}$. To encode equivalence under fiber-preserving transformations (i.e. taking in account the Lie equations (1.1)) we must have

$$
\varphi^{*} d \bar{x}=a_{4} d x
$$

for a certain function $a_{4}: \mathrm{J}^{1} \rightarrow \mathbb{C}$. Summarizing: two second order differential equations $\mathcal{E}_{f}$ and $\mathcal{E}_{\bar{f}}$ are equivalent under a fiber-preserving transformation $\varphi$ if and only if

$$
\varphi^{*}\left(\begin{array}{c}
d \bar{y}-\bar{p} d \bar{x} \\
d \bar{p}-\bar{f}(\bar{x}, \bar{y}, \bar{p}) d \bar{x} \\
d \bar{x}
\end{array}\right)=\left(\begin{array}{ccc}
a_{1} & 0 & 0 \\
a_{2} & a_{3} & 0 \\
0 & 0 & a_{4}
\end{array}\right)\left(\begin{array}{c}
d y-p d x \\
d p-f(x, y, p) d x \\
d x
\end{array}\right) .
$$

For this problem, Cartan's equivalence method ( $(\underline{\operatorname{Olv} 95})$ gives three fundamental invariants

$$
\left\{\begin{array}{l}
I_{3}=-\frac{f_{p p p} a_{4}}{2 a_{1}^{2}} \\
I_{2}=\frac{f_{y p}-D_{x} f_{p p}}{2 a_{1} a_{4}} \\
I_{1}=\frac{\left(2 f_{y y}-D_{x} f_{y p}-f_{p p} f_{y}+f_{y p} f_{p}\right) a_{1}+\left(-f_{y p}+D_{x} f_{p p}\right) a_{4} a_{2}}{2 a_{1}^{2} a_{4}^{2}}
\end{array}\right.
$$

and six invariant derivations defined on certain manifold $\tilde{M}$, fibred over $\mathrm{J}^{1}$, with local coordinates de $\left(x, y, p, a_{1}, a_{2}, a_{4}\right)$. Here, $D_{x}=\partial_{x}+p \partial_{y}+f \partial_{p}$ is Cartan vector field.

When specializing on the Painlevé equations, the two fundamental invariants $I_{2}$ and $I_{3}$ vanish. On this splitting branch, the application of Jaccobi identity to the final structure equations shows that among the six invariant derivations only two can produce new invariants. These two derivations are

$$
\left\{\begin{array}{l}
X_{1}=\frac{1}{a_{1}} \partial_{y}-\frac{a_{2} a_{4}}{a_{1}{ }^{2}} \partial_{p}-\frac{1}{2} f_{p p} \partial_{a_{1}}-\frac{1}{2} \frac{f_{p y}}{a_{4}} \partial_{a_{2}} \\
X_{3}=\frac{1}{a_{4}} \partial_{x}+\frac{p}{a_{4}} \partial_{y}+\frac{f}{a_{4}} \partial_{p}+a_{2} \partial_{a_{1}}-\frac{f_{y} a_{1}}{a_{4}{ }^{2}} \partial_{a_{2}}+\frac{2 a_{2} a_{4}+f_{p} a_{1}}{a_{1}} \partial_{a_{4}} .
\end{array}\right.
$$

Notation 1 In the sequel, $I_{1 ; j \cdots k}$ denotes the differential invariant $X_{k} \cdots X_{j}\left(I_{1}\right)$. For instance, the invariant $I_{1 ; 33}$ is obtained by differentiating twice the fundamental invariant $I_{1}$ with respect to invariant derivation $X_{3}$.

\section{The first Painlevé equation $y^{\prime \prime}=6 y^{2}+x$}

Since the associated fiber-preserving symmetry Lie pseudo-group is zero dimensional, this justify the following lemma:

Lemma 1 The specialization of the invariants

$$
I_{1}, I_{1 ; 3}, I_{1 ; 33}, \frac{I_{1 ; 333}}{I_{1 ; 33}}, \frac{I_{1 ; 3333}}{I_{1 ; 33}}-\frac{43}{120} I_{1 ; 33}, \frac{I_{1 ; 33333}}{I_{1 ; 33}}-\frac{5}{4} I_{1 ; 33}
$$

on the first Painlevé equation gives six invariants functionally independent defined on $\tilde{M}$.

The problem with the above invariants is that they do depend on extra parameters $a_{1}, a_{2}$ and $a_{4}$. Fortunately, in our zero-dimensional case, we can normalize (e.i. eliminate) these parameters by setting

$$
I_{1}=-12, I_{1 ; 3}=0, \frac{I_{1 ; 333}}{I_{1 ; 33}}=1
$$

Now substituting the values of the parameters in the remaining invariants give us, due again to our zero-dimensional case, three functionally independent invariants now do not depending on the extra parameters. 
Writing the equality of the invariants and simplifying the obtained system, by computing a characteristic set ( Kol73; BLOP95), gives an algebraic transformation of degree 5

$$
\left\{\begin{array}{l}
\bar{p}=129600 \frac{\left(5 I_{1 ; 33}{ }^{2}+4 I_{1 ; 33333}\right)}{I_{1 ; 33}{ }^{3}} \bar{y}^{4} \\
\bar{x}=-6 \frac{\left(120 I_{1 ; 3333}+43 I_{1 ; 33}{ }^{2}\right)}{I_{1 ; 33}^{2}} \bar{y}^{2}, \\
\bar{y}^{5}=-\frac{1}{23328000} \frac{I_{1 ; 33}{ }^{5}}{\left(5 I_{1 ; 33}{ }^{2}+4 I_{1 ; 3333}\right)^{2}} .
\end{array}\right.
$$

In these formulae the invariants are normalized using (3.1), that is, do not depending on the extra parameters. According to (ii) of the introduction and (1.2), we have

Theorem 1 A second order differential equation $\mathcal{E}_{f}$ is equivalent to the first Painlevé equation by a fiberpreserving transformation if and only if this transformation is given by (3.2) and the normalization (3.1).

Let us explain how Theorem 1 can be used in practice. Consider the following equations

$$
y^{\prime \prime}=c \frac{y^{\prime 2}}{y}+\frac{1}{y}\left(y^{4}+x\right),
$$

and

$$
y^{\prime \prime}=c \frac{y^{\prime 2}}{y}+y\left(y^{4}+x\right) .
$$

The question is to determine the values of the parameter $c$ for which the above equations can be mapped to the first Painlevé equation (and compute the equivalence transformation when the equivalence holds).

First of all, the fact that the derived invariants $I_{1 ; 1}$ vanishes on the first Painlevé equation restricts the possible values of $c$ to $\{-1,3\}$ for the first equation and to $\{-3,5\}$ for the second equation.

The second step is to specialize (3.2) on the given equation to obtain transformation candidates. In step 3, we have to check whether the pullback of the first Painlevé equation w.r.t these candidates is exactly the considered equation.

In the case of equation (3.3), the specialization yields

$$
\left\{\begin{array}{l}
\bar{p}=36 \frac{\bar{y}^{4} p}{y^{7}} \\
\bar{x}=6 \frac{\bar{y}^{2} x}{y^{4}} \\
\bar{y}^{5}=\frac{1}{108} y^{10}
\end{array}\right.
$$

for $c=-1$ and

$$
\left\{\begin{aligned}
\bar{p} & =-864 \frac{\bar{y}^{4} y^{5}\left(625 x^{5}-2079\right)\left(-25 y x^{3}+250 p x^{4}+21 y^{3}\right)}{\left(50 x^{3}+3 y^{2}\right)^{4}}, \\
\bar{x} & =6 \frac{\left(2500 x^{5}-891\right) y^{4} \bar{y}^{2}}{\left(50 x^{3}+3 y^{2}\right)^{2}}, \\
\bar{y}^{5} & =-\frac{1}{31104} \frac{\left(50 x^{3}+3 y^{2}\right)^{5}}{y^{10}\left(625 x^{5}-2079\right)^{2}}
\end{aligned}\right.
$$

for $c=3$. The third steps shows that the equivalence holds only for $c=-1$ and the equivalence transformation is (3.5). We can also deduce, according to (ii) in the introduction, that the cardinal of the fiber-preserving (point) symmetry group of the equation (3.3) with $c=-1$ is equal to 10 .

The same calculations show that equation (3.4) can not be mapped to the first Painlevé equation. In particular, we have a division by zero error in step 2 for $c=5$. Warning: This error doesn't mean that the method failed. In fact it is part of the method and implies that no equivalence transformation does exist.

Time estimates are given in tables bellow where $P_{1}$ refers to the first Painlevé equation. 


\begin{tabular}{|c|c|c|}
\hline & Computation of transformation candidates & Checking equivalence with $P_{1}$ \\
\hline$c=-1$ & 0.15 & (yes) 0.04 \\
\hline$c=3$ & 2.13 & (no) 0.13 \\
\hline
\end{tabular}

Table 1. Time estimates (in seconds) for $y^{\prime \prime}=c \frac{y^{\prime 2}}{y}+\frac{1}{y}\left(y^{4}+x\right)$

\begin{tabular}{|c|c|c|}
\hline & Computation of transformation candidates & Checking equivalence with $P_{1}$ \\
\hline$c=-3$ & 0.35 & (no) 0.03 \\
\hline$c=5$ & division by zero error & (no) 0.00 \\
\hline
\end{tabular}

Table 2. Time estimates (in seconds) for $y^{\prime \prime}=c \frac{y^{\prime 2}}{y}+y\left(y^{4}+x\right)$

\section{The second Painlevé equation $y^{\prime \prime}=2 y^{3}+x y+\alpha$}

Again, due to the zero-dimensionality, there exists seven invariants defined on the manifold of local coordinates $\left(x, y, p, a_{1}, a_{2}, a_{4}, \alpha\right)$ such that when specialized, on Painlevé two, they give exactly seven functionally independent functions. For instance, one can take the invariants $I_{1}, I_{1 ; 3}, I_{1 ; 31}, I_{1 ; 33}, I_{1 ; 331}, I_{1 ; 3331}$ and $I_{1 ; 33311}$. We normalize $a_{1}, a_{2}$ and $a_{4}$ by setting

$$
I_{1}=-12, I_{1 ; 3}=-12, I_{1 ; 31}=0,
$$

and as in the previous section, we obtain

$$
\left\{\begin{aligned}
\bar{p}= & \frac{1}{6}\left(\frac{I_{1 ; 33311}\left(I_{1 ; 3331}+4032\right)}{I_{1 ; 33311} I_{1 ; 33}-3096576-4032 I_{1 ; 331}}\right) \bar{y}^{2} \bar{\alpha} \\
\bar{x}= & -\left(16+\frac{1}{72} I_{1 ; 331}\right) \bar{y}^{2}, \\
\bar{y}^{3}= & 48384 \frac{\bar{\alpha}}{I_{1 ; 33311} I_{1 ; 33}-3096576-4032 I_{1 ; 331}}, \\
\bar{\alpha}^{2}= & -\frac{1}{112 I_{1 ; 33311}\left(16257024+8064 I_{1 ; 3331}+I_{1 ; 3331}{ }^{2}\right)}\left(I_{1 ; 33311^{2}} I_{1 ; 33}{ }^{2}\right. \\
& -8064 I_{1 ; 33311} I_{1 ; 33} I_{1 ; 331}-6193152 I_{1 ; 33311} I_{1 ; 33} \\
& \left.+9588782923776+24970788864 I_{1 ; 331}+16257024 I_{1 ; 331}{ }^{2}\right) .
\end{aligned}\right.
$$

when $\alpha \neq 0$ and

$$
\left\{\begin{aligned}
\bar{p} & =\frac{1}{290304} y^{5} I_{1 ; 33311}\left(4032+I_{1 ; 3331}\right), \\
x & =-\frac{1}{72} y^{2}\left(1152+I_{1 ; 331}\right), \\
y^{6} & =-20901888 \frac{1}{I_{1 ; 33311}\left(4032+I_{1 ; 3331}\right)^{2}},
\end{aligned}\right.
$$

when $\alpha=0$. The comparison with the symmetry pseudo-groups (1.3) and (1.4) proves

Theorem 2 A second order differential equation can be mapped to the second Painlevé equation $y^{\prime \prime}=$ $2 y^{3}+y x+\alpha$ by a fiber-preserving transformation if and only if this transformation is given by (4.2) if $\alpha \neq 0$ and by (4.3) otherwise with the normalization (4.1) in both cases.

Let us remark that (4.3) can be obtained from (4.2) (as well as (1.4) from (1.3)) by eliminating the $\bar{\alpha}$ and taking in account the functional dependence between the invariants resulting from $\bar{\alpha}=0$. Nevertheless, it is more safe to separate the two cases $(\alpha \neq 0$ and $\alpha=0)$.

\section{Equivalence under point transformation}

The equivalence problem under the more general point transformations naturally arises since Painlevé equations belong to the class of equations of the form

$$
y^{\prime \prime}=A(x, y)+B(x, y) y^{\prime}+C(x, y) y^{\prime 2}+D(x, y) y^{\prime 3}
$$


which is invariant under point transformations. In this case our starting Pfaffian system is

$$
\varphi^{*}\left(\begin{array}{c}
d \bar{y}-\bar{p} d \bar{x} \\
d \bar{p}-\bar{f}(\bar{x}, \bar{y}, \bar{p}) d \bar{x} \\
d \bar{x}
\end{array}\right)=\left(\begin{array}{ccc}
a_{1} & 0 & 0 \\
a_{2} & a_{3} & 0 \\
a_{4} & 0 & a_{5}
\end{array}\right)\left(\begin{array}{c}
d y-p d x \\
d p-f(x, y, p) d x \\
d x
\end{array}\right)
$$

for which we normalize $a_{3}$ and prolong to obtain involution and four fundamental invariants defined on 8-dimensional manifold. For the above class, only two invariants are not identically zero

$$
\begin{aligned}
K_{1}= & \left(6 f_{y y}-4 D_{x} f_{y p}+D_{x}{ }^{2} f_{p p}-3 f_{y} f_{p p}+4 f_{y p} f_{p}-D_{x} f_{p p} f_{p}\right) /\left(a_{1} a_{5}{ }^{2}\right), \\
K_{2}= & \left(2 f_{y} f_{p p p} a_{5}+4 f_{y p} f_{p} a_{4}-D_{x} f_{p p} f_{p} a_{4}-3 f_{y} f_{p p} a_{4}-a_{5} f_{p p} f_{y p}+a_{5} f_{p p} D_{x} f_{p p}+6 a_{4} f_{y y}\right. \\
& +a_{4} D_{x} D_{x} f_{p p}-a_{5} D_{x} f_{p p p} f_{p}-a_{5} f_{p p p} D_{x} f_{p}-4 a_{4} D_{x} f_{y p}-2 f_{y y p} a_{5}+2 a_{5} D_{x} f_{y p p} \\
& \left.-a_{5} D_{x} D_{x} f_{p p p}\right) /\left(a_{5}{ }^{2} a_{1}{ }^{2}\right) .
\end{aligned}
$$

As in the fiber-preserving case, only two invariant derivations $X_{1}$ and $X_{3}$ (one page long) are needed.

Theorem 3 A second order ordinary differential equation $y^{\prime \prime}=f\left(x, y, y^{\prime}\right)$ is equivalent

(i) to the first Painlevé equation $y^{\prime \prime}=6 y^{2}+x$ under a point transformation if and only if this transformation is given by

$$
\left\{\begin{aligned}
\bar{p} & =\frac{5}{1056} \frac{\left(2^{15} 3^{5} 11^{3} K_{1 ; 33333}+K_{\left.1 ; 33313^{3}\right)}\right.}{K_{1 ; 33313}{ }^{2}} \bar{y}^{4} \\
\bar{x} & =-6 \frac{\left(2^{9} 3^{3} 511^{2} K_{1 ; 3333}+43 K_{\left.1 ; 33313^{2}\right)}\right.}{K_{1 ; 33313^{2}}} \bar{y}^{2} \\
y^{5} & =-\frac{88}{375} \frac{K_{1 ; 33313}{ }^{5}}{\left(2^{15} 3^{5} 11^{3} K_{1 ; 33333}+K_{1 ; 33313^{3}}\right)^{2}}
\end{aligned}\right.
$$

with the normalization

$$
K_{1}=-12, K_{2}=0, K_{1 ; 1}=0, K_{1 ; 3}=0, K_{1 ; 33} / K_{1 ; 333}=720 .
$$

(ii) to the second Painlevé equation $y^{\prime \prime}=2 y^{3}+x y+\alpha$ under a point transformation if and only if this transformation is given by

$$
\left\{\begin{aligned}
\bar{p} & =-\frac{1}{18} \frac{K_{2 ; 3}\left(15 K_{2 ; 3} K_{1 ; 33}-216000+4032 K_{2 ; 3}-450 K_{1 ; 331}-50 K_{2 ; 3} K_{1 ; 333}\right)}{25 K_{2 ; 3} K_{1 ; 33}-115200+1728 K_{2 ; 3}-150 K_{1 ; 331}} \bar{y}^{2} \\
\bar{x} & =\frac{1}{3600}\left(25 K_{2 ; 3} K_{1 ; 33}+336 K_{2 ; 3}-57600-50 K_{1 ; 331}\right) \bar{y}^{2} \\
\bar{y}^{3} & =-1800 \frac{\bar{\alpha}}{25 K_{2 ; 3} K_{1 ; 33}-115200+1728 K_{2 ; 3}-150 K_{1 ; 331}}, \\
\bar{\alpha}^{2} & =-108 \frac{\left(25 K_{2 ; 3} K_{1 ; 33}-115200+1728 K_{2 ; 3}-150 K_{1 ; 331}\right)^{2}}{K_{2 ; 3}\left(15 K_{2 ; 3} K_{1 ; 33}-216000+4032 K_{2 ; 3}-450 K_{1 ; 331}-50 K_{2 ; 3} K_{1 ; 333}\right)^{2}}
\end{aligned}\right.
$$

when $\alpha \neq 0$ and

$$
\left\{\begin{aligned}
\bar{p} & =-\frac{1}{16200} K_{2 ; 3}\left(576 K_{2 ; 3}+25 K_{2 ; 3} K_{1 ; 333}+30 K_{2 ; 3} K_{1 ; 33}-64800\right) \bar{y}^{5} \\
\bar{x} & =\frac{1}{1080}\left(5 K_{2 ; 3} K_{1 ; 33}-5760-72 K_{2 ; 3}\right) \bar{y}^{2} \\
\bar{y}^{6} & =-\frac{87480000}{K_{2 ; 3}\left(576 K_{2 ; 3}+25 K_{2 ; 3} K_{1 ; 333}+30 K_{2 ; 3} K_{1 ; 33}-64800\right)^{2}}
\end{aligned}\right.
$$

when $\alpha=0$, with the normalization

$$
K_{1}=-12, K_{2}=0, K_{1 ; 1}=0, K_{1 ; 3}=0, K_{2 ; 3} / K_{1 ; 31}=-5 / 24 .
$$


Example. Let us terminate with considering the equivalence of the two equations (3.3) and (3.4) with the second Painlevé equation under point transformations. Here, computations are done with arbitrary $c$.

The equation (3.3): The specialization of (5.3) on this equation yields (after 0.512 seconds) a transformation candidate depending on $c$ and which is too long to include in this paper. The variable $\bar{x}$ doesn't depend on $p$ in only tow cases $c \in\{-1,3\}$ and this two values return a division by zero error when computing the others components. The same thing happens with the specialization of (5.3) on (3.3). Thus, equation (3.3) can't be equivalent to the second Painlevé equation under point transformations.

The equation (3.4): The specialization of (5.2) on this equation gives the following transformation (in 1.11 seconds)

$$
\left\{\begin{aligned}
\bar{p}= & \frac{1}{36} \frac{(c+3)(c-2)^{2} p}{(1+c)(c-5) y^{12}} \times\left(9 y^{3} c+66 y^{6} p+\cdots+27 y^{3}\right) \bar{\alpha} \bar{y}^{2} \\
\bar{x}= & \frac{2}{3} \frac{\left(-27 y^{6}+3 y^{2} x c-2 c^{2} p^{2}-24 y^{6} c+3 y^{6} c^{2}+5 c p^{2}-18 y^{2} x+6 p^{2}-c^{3} p^{2}+3 y^{2} x c^{2}\right)}{(c-5) y^{6}} \bar{y}^{2} \\
\bar{y}^{3}= & \frac{1}{16} \frac{(c-5)}{1+c} \bar{\alpha} \\
\bar{\alpha}^{2}= & 1728 \frac{(5-c)(1+c)^{2}}{(c+3)(c-2)^{2}} y^{18} \times\left(-9 y^{3} c-66 y^{6} p+54 y^{2} p x+18 y^{6} c^{2} p-48 y^{6} p c+18 y^{2} x c^{2} p\right. \\
& \left.+2 c^{3} p^{3}-2 p^{3} c^{2}+72 y^{2} p x c-34 p^{3} c-30 p^{3}-27 y^{3}\right)^{-2} .
\end{aligned}\right.
$$

For the particular values of $c$ for which $\bar{x}$ does not depend on $p$ we obtain division by zero errors when computing the others components and then the equation (3.4) can not be mapped to Painlevé two with $\alpha \neq 0$. However, the specialization of (5.3) on (3.4) gives

$$
\left\{\begin{aligned}
\bar{p}= & -\frac{4}{9} \frac{(c-2)^{2} p(c+3)\left(18 x y^{2} p c^{2}-90 p y^{6}+\cdots+18 y^{6} p c^{2}\right)}{(c-5)^{2} y^{12}} \bar{y}^{5} \\
\bar{x}= & \frac{2}{3} \frac{\left(-23 y^{6}+3 y^{2} x c-2 c^{2} p^{2}-20 y^{6} c+3 y^{6} c^{2}+5 c p^{2}-18 y^{2} x+6 p^{2}-c^{3} p^{2}+3 y^{2} x c^{2}\right)}{(c-5) y^{6}} \bar{y}^{2} \\
\bar{y}^{6}= & -\frac{27}{4} \frac{(c-5)^{3}}{(c+3)(c-2)^{2}} y^{18} \times\left(18 x y^{2} p c^{2}-90 p y^{6}-9 y^{3} c+54 p x y^{2}-27 y^{3}+72 p c x y^{2}-34 p^{3} c\right. \\
& \left.-72 p c y^{6}+2 c^{3} p^{3}-30 p^{3}-2 p^{3} c^{2}+18 y^{6} p c^{2}\right)^{-2}
\end{aligned}\right.
$$

which is point transformation only when $c=-1$. In this case, the resulting transformation is

$$
\bar{p}=4 \frac{\bar{y}^{5} p}{y^{9}}, \bar{x}=2 \frac{\bar{y}^{2} x}{y^{4}}, \bar{y}^{6}=1 / 4 y^{12}
$$

and this maps the equation (3.4) to Painlevé two $y^{\prime \prime}=2 y^{3}+x y$ (with $\alpha=0$ ).

\section{Acknowledgment}

The author wishes to thank Michel Petitot for fruitful discussions, Samuel Vidal for encouragements, Pavel Exner for financial support and anonymous referees for their valuable comments.

\section{References}

[BLOP95] F. Boulier, D. Lazard, F. Ollivier, and M. Petitot, Representation for the radical of a finitely generated differential ideal, proc. ISSAC'95 (Montréal, Canada), 1995, pp. 158-166.

[Car24] E. Cartan, Sur les variétés à connexion projective, Bull. Soc. Math. France 52 (1924), 205-241. MR MR1504846 
[CM08] Robert M. Conte and Micheline Musette, The Painlevé handbook., Dordrecht: Springer. xxiv, 256 p., 2008.

[DP07] R. Dridi and M. Petitot, Towards a new ode solver based on cartan's equivalence method, ISSAC '07: Proceedings of the 2007 international symposium on Symbolic and algebraic computation (New York, NY, USA), ACM Press, 2007, pp. 135-142.

[HD02] J. Hietarinta and V. Dryuma, Is my ode a painlevé equation in disguise?, J. Nonlinear Math. Phys. 9 (2002), 467-74.

[KLS85] N. Kamran, K. G. Lamb, and W. F. Shadwick, The local equivalence problem for $d^{2} y / d x^{2}=$ $F(x, y, d y / d x)$ and the Painlevé transcendents, J. Differential Geom. 22 (1985), no. 2, 139-150. MR MR834275 (88f:58004)

[Kol73] E.R. Kolchin, Differential algebra and algebraic groups, Academic press, New-York and london, 1973.

[Olv93] P. J. Olver, Applications of Lie groups to differential equations, Graduate Texts in Mathematics, Springer-Verlag, 1993.

[Olv95] _ Equivalence, invariants, and symmetry, Cambridge University Press, Cambridge, 1995. MR MR1337276 (96i:58005) 